\title{
Analysis of non-protein amino acids as specific markers of low density lipoprotein apolipoprotein B-100 oxidation in human atherosclerotic lesions: the use of $N(O)$-ethoxycarbonyl trifluoroethyl ester derivatives and GC-MS ${ }^{1}$
}

\author{
Jens Pietzsch $^{\mathrm{a}, *}$, Ralf Bergmann ${ }^{\mathrm{a}}$ and Steffi Kopprasch ${ }^{\mathrm{b}}$ \\ ${ }^{a}$ Institute of Bioinorganic and Radiopharmaceutical Chemistry, Research Center Rossendorf Dresden, \\ P.O. Box 5101 19, D-01314 Dresden, Germany \\ ${ }^{\mathrm{b}}$ Department of Internal Medicine 3, Carl Gustav Carus Medical School, University of Technology \\ Dresden, Fetscherstraße 74, D-01307 Dresden, Germany
}

\begin{abstract}
Oxidative modification of proteins can interfere with critical cellular functions, and is widely regarded as a crucial event in the pathogenesis of various diseases ranging from rheumatoid arthritis to atherosclerosis and cancer. In this line, a new GC-MS methodology using $N(O)$-ethoxycarbonyl trifluoroethyl amino acid esters $\left(E C E E-\mathrm{F}_{3}\right)$ for rapid and sensitive determination of 3-chlorotyrosine, 5-hydroxy-2-aminovaleric acid (HAVA), and 6-hydroxy-2-aminocaproic acid (HACA) in proteins has been developed. 3-Chlorotyrosine is a highly specific marker of myeloperoxidase catalyzed protein oxidation, whereas $\gamma$-glutamyl semialdehyde ( $\gamma \mathrm{GSA}$ ) and $\alpha$-aminoadipyl semialdehyde ( $\alpha \mathrm{ASA}$ ), which by reduction form HAVA and HACA, respectively, are specifically formed by metal catalyzed oxidation processes. ECEE- $\mathrm{F}_{3}$ derivatives are formed by the unlabored reaction of amino acids with ethylchloroformate plus trifluoroethanol plus pyridine. The key steps of the methodology employed are (i) enzymatic hydrolysis of target proteins to prevent decomposition of oxidation products during hydrolysis and (ii) an uniquely rapid derivatization of amino acids completing sample preparation for GC within a few minutes in aqueous solution at room temperature. The use of these stable products of protein amino acid side chain oxidation as potential markers for assessing oxidative damage in LDL apoB-100 recovered from human aortic vascular lesions is demonstrated. These observations provide quantitative chemical evidence for metal catalyzed oxidative processes in the human artery wall.
\end{abstract}

Keywords: Amino acids, atherosclerosis, low density lipoprotein, protein oxidation, gas chromatography-mass spectrometry

\footnotetext{
${ }^{1}$ A part of this report has been presented at the 2nd International Conference on Biomedical Spectroscopy, London, UK, 5-8 July 2003.

*Corresponding author: Dr. Jens Pietzsch, Pathological Biochemistry Group, Department Positron Emission Tomography, Institute of Bioinorganic and Radiopharmaceutical Chemistry, Research Center Rossendorf, P.O. Box 5101 19, D-01314 Dresden, Germany. Fax: +49 351260 3661; E-mail: j.pietzsch@fz-rossendorf.de.
} 


\section{Abbreviations}

$\alpha$ ASA, $\alpha$-aminoadipyl semialdehyde; apo, apolipoprotein; $\gamma \mathrm{GSA}, \gamma$-glutamyl semialdehyde; HACA, 6-hydroxy-2-aminocaproic acid; HAVA, 5-hydroxy-2-aminovaleric acid; LDL, low density lipoprotein; MPO, myeloperoxidase.

\section{Introduction}

Oxidative modification of proteins can interfere with critical cellular functions and affect enzyme function and cellular signaling, and is widely regarded as a crucial event in the pathogenesis of various diseases ranging from atherosclerosis to rheumatoid arthritis and cancer [1,2]. In this line, oxidative modification of apolipoprotein (apo) B-100, the major protein of low density lipoprotein (LDL), is thought to finally result in the formation of new epitopes that are specifically recognized by scavenger receptors followed by an excessive uptake and accumulation of LDL particles in macrophages and vascular smooth muscle cells that can lead to foam cell formation [3]. The latter appears to be the earliest morphologic substrate of atherogenesis [4]. However, the nature of specific modifications of protein amino acid side chain residues, thus altering their cellular functions, is a current matter of debate $[1,2]$. One potential pathway for direct oxidation of apoB-100 involves myeloperoxidase (MPO), that finally generates highly specific oxidation products, such as 3-chlorotyrosine [1,5]. At physiological plasma concentrations of hydrogen peroxide and chloride the heme protein MPO generates the highly reactive hypochlorite ion $\left({ }^{-} \mathrm{OCl}\right)$. Proteins exposed to $\mathrm{MPO} / \mathrm{H}_{2} \mathrm{O}_{2} / \mathrm{Cl}^{-}$, as well as to reagent $\mathrm{HOCl}$, undergo chlorination of their tyrosine side chain residues [6,7]. In humans the generation of $\mathrm{HOCl}$ by phagocytic leukocytes is important in host defense against invading pathogens [5,7]. On the other hand, oxidant production by leukocytes is also potentially deleterious to endogenous proteins [5,7].

Furthermore, there is effectual evidence supporting the hypothesis that among important mechanisms of protein oxidation are also metal catalyzed processes [2]. These processes involve binding of either free or, physiologically more relevant, complexed (porphyrin-bound) redox-active iron $\left(\mathrm{Fe}^{2+} / \mathrm{Fe}^{3+}\right)$ to discrete binding sites of LDL and apoB-100, respectively, thus forming centers for redox cycling, e.g., perferryl cycles via $\left[\mathrm{Fe}^{\mathrm{IV}}=\mathrm{O}\right]$ and $\left[\mathrm{Fe}^{\mathrm{V}}-\mathrm{OH}\right]$ of heme iron and hydroperoxides, and repeated radical production [8]. Two major products of transition metal catalyzed protein oxidation are $\gamma$-glutamyl semialdehyde ( $\gamma \mathrm{GSA}$ ) and $\alpha$-aminoadipyl semialdehyde ( $\alpha$ ASA) [9]. By reduction $\gamma$ GSA forms 5-hydroxy2-aminovaleric acid (HAVA) and $\alpha$ ASA forms 6-hydroxy-2-aminocaproic acid (HACA), respectively [10-12]. Experimental evidence reveals $\gamma \mathrm{GSA}$ and $\alpha \mathrm{ASA}$ to be the major constituents of the total protein carbonyl content in various model proteins subjected to metal catalyzed oxidation in vitro $[9,10]$. It has been suggested that oxidation of apoB- 100 by both $\mathrm{MPO} / \mathrm{HOCl}$ and transition metals is involved in atherogenesis [1]. The objective of this report is the investigation of the feasibility of the use of $N(O)$-ethoxycarbonyl trifluoroethyl ester $\left(\mathrm{ECEE}^{\left.-\mathrm{F}_{3}\right)}\right.$ derivatives and gas chromatography/mass spectrometry (GC/MS) to quantify low-abundance HAVA, HACA, and 3-chlorotyrosine residues in isolated apoB-100. The analysis of these fluorinated derivatives for quantitative analysis of amino acids by chemical ionization mass spectrometry has been described by Moini and colleagues [13,14]. More recently, the use of ECEE- $F_{3}$ derivatives for analysis of protein and non-protein amino acids by standard electron impact ionization (EI) mass spectrometry has been described by us $[6,15]$. In the present study we demonstrate that levels of HAVA, HACA, and 3-chlorotyrosine are markedly elevated in LDL apoB-100 recovered from human aortic vascular lesions. These observations provide quantitative chemical and clinical evidence for both MPO and metal catalyzed oxidative processes in the human artery wall. 


\section{Materials and methods}

\subsection{Chemicals}

Ethyl chloroformate was obtained from Fluka (Buchs, Switzerland). L-Amino acids, L-norleucine, L-p-chlorophenylalanine (internal standards), 2,2,2-trifluoroethanol, and nonspecific protease Type XIV (from Streptomyces griseus), were purchased from Sigma (St Louis, MO, USA). All other chemicals were obtained from Sigma and BioRad (Richmond, CA, USA).

\subsection{Lipoprotein isolation}

Lesion LDL were isolated from the intima of normal and atherosclerotic specimen of human thoracic aortas obtained at necropsy within $10 \mathrm{~h}$ of sudden death (20 male accident victims, aged 26 to 45 years; no signs of severe acute diseases). Normal and atherosclerotic tissue (fatty streaks, intermediate, and advanced plaques) was classified according to the criteria of the Pathobiological Determinants of Atherosclerosis in Youth Study [16]. Samples were processed in subdued light to prevent photooxidation. Aortic intima was dissected from the medium and homogenized for $2 \mathrm{~h}$ at $4{ }^{\circ} \mathrm{C}(30 \mathrm{mg}$ wet tissue $/ \mathrm{ml}$ phosphate buffered saline, $\mathrm{pH}$ 7.2). All buffers and solutions were degassed and stored under argon. Furthermore, all buffers and solutions were supplemented with $100 \mu \mathrm{M}$ diethylenetriaminepentaacetic acid (DTPA), $100 \mu \mathrm{M}$ butylated hydroxyanisole (BHA), and $100 \mu \mathrm{M}$ 3-amino-1,2,4-triazole. Lesion LDL (density $1.006-1.063 \mathrm{~g} / \mathrm{ml}$ ) were then isolated by very fast ultracentrifugation at $120,000 \mathrm{rev} . / \mathrm{min}$ corresponding to $625,000 \times g$ with a run time of $100 \mathrm{~min}$ at $18^{\circ} \mathrm{C}$ [17]. Delipidation and reduction of LDL, respectively, as well as enzymatic hydrolysis of apoB-100 using nonspecific protease Type XIV were performed as previously described [10].

\subsection{GC-MS analysis}

The free amino acids from protein hydrolysates were isolated by cation exchange chromatography using Dowex AG-50W-X4 (H+, 100-200 mesh) resin. The purified and dried protein amino acids were derivatized to their ECEE- $\mathrm{F}_{3}$ derivatives and analyzed following the protocol described previously by us [15]. In brief, amino acids in residues (not more than $50 \mu \mathrm{g}$ in total) were treated with $100 \mu \mathrm{l}$ of trifluoroethanol/pyridine in a volume ratio $80: 20$. Then $10 \mu \mathrm{l}$ of ethyl chloroformate were added and mixed by shaking the tube gently for 30 seconds. The derivatives were extracted with $150 \mu \mathrm{l}$ of chloroform containing $2 \%$ ethyl chloroformate. An aliquot was taken from the chloroform layer and injected. The analysis was conducted using a model 5890II GC/5989A MS-Engine equipped with a $25 \mathrm{~m} \times$ $0.20 \mathrm{~mm}$ HP-5 capillary column (5\% diphenyl-95\% dimethylpolysiloxane, $0.33 \mu \mathrm{m}$; Hewlett-Packard, Palo Alto, CA, USA). The GC conditions were as follows: carrier gas, helium; column head pressure, $70 \mathrm{kPa}$; injector temperature, $250^{\circ} \mathrm{C}$; oven temperature gradient program, $110^{\circ} \mathrm{C}$ increased to $320^{\circ} \mathrm{C}$ at $25^{\circ} \mathrm{C} / \mathrm{min}$; interface temperature $250^{\circ} \mathrm{C}$. EI-MS conditions were as follows: source temperature, $250^{\circ} \mathrm{C}$; analyzer temperature, $120^{\circ} \mathrm{C}$; electron energy, $70 \mathrm{eV}$. All samples were run in triplicate. Under the mass spectrometric conditions employed the most abundant and characteristic ions of the HAVA, HACA, and 3-chlorotyrosine ECEE- $\mathrm{F}_{3}$ derivatives were the $[\mathrm{M}-127]^{+}$ion (base peak) resulting from the loss of ${ }^{\bullet} \mathrm{CO}_{2} \mathrm{CH}_{2} \mathrm{CF}_{3}$ and the $[\mathrm{M}-89]^{+\bullet}$ ion from the loss of $\mathrm{NH}_{2} \mathrm{CO}_{2} \mathrm{Et}$ from the corresponding molecular ions $\left([\mathrm{M}]^{+\bullet}\right.$ ), respectively $[6,10,18]$. Diagnostically useful ions for HAVA (at $\mathrm{m} / z 160$ and $\mathrm{m} / z$ 198), HACA (at $m / z 174$ and $m / z 212$ ), 3-chlorotyrosine (at $m / z 314$ and $m / z 352$ ), norleucine (at $m / z$ 158 and $m / z$ 196), and L-p-chlorophenylalanine (at $m / z 226$ and $m / z$ 264) were monitored in the 
selected ion monitoring (SIM) mode with a dwell time of 25 or $50 \mathrm{~ms}$ for each ion under the control of the HP-ChemStation data system [6,18]. Data acquisition was delayed until 2 min after injection. For quantification of HAVA, HACA, and 3-chlorotyrosine, calibration curves were obtained by plotting the peak area ratio for each characteristic oxidation marker - internal standard ion pair (HAVA and HACA, respectively, vs. L-norleucine; 3-chlorotyrosine $v s$. L-p-chlorophenylalanine) $v s$. the mass ratio of the analytes and the internal standard, respectively. Calibration curves were analyzed by unweighted least-squares linear regression analysis and were found to be linear over the range studied $\left(2-600 \mathrm{nM} ; R^{2}>0.99\right)$ and of good quality (intraassay CV, $<4.5 \%$; interassay CV, $<6.1 \%$ ). Signal reproducibility was found to be less than $8 \%$ variation in the peak areas being observed for the $\left[\mathrm{M}-\mathrm{NH}_{2} \mathrm{CO}_{2} \mathrm{Et}\right]^{+\bullet}$ and $\left[\mathrm{M}-\mathrm{CO}_{2} \mathrm{CH}_{2} \mathrm{CF}_{3}\right]^{+}$ions, respectively. The limit of determination of all analytes

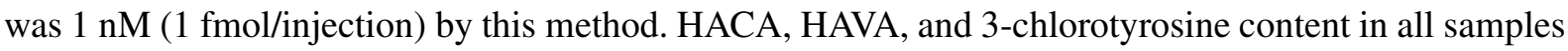
is expressed as $\mathrm{mol} / \mathrm{mol}$ apoB-100.

\section{Results and discussion}

Figures 1 to 3 show the content of HAVA, HACA, and 3-chlorotyrosine in the intima from various stages of lesion evolution as determined by sensitive and specific GC-MS analysis using amino acid ECEE- $F_{3}$ derivatives. The key steps of this methodology were: (i) the use of very fast ultracentrifugation (VFU) for isolation of native LDL free of contaminating albumin; (ii) enzymatic hydrolysis of apoB-100 using nonspecific protease Type XIV to prevent decomposition of oxidation products during hydrolysis; (iii) an uniquely rapid derivatization of amino acids (except arginine, owing to the underivatized imino group of its guanidine moiety) completing sample preparation for GC within a few minutes in aqueous solution at room temperature; and (iv) the use of standard EI mode that is easy to maintain and reproduce compared with chemical ionization mode $[6,15,18]$.

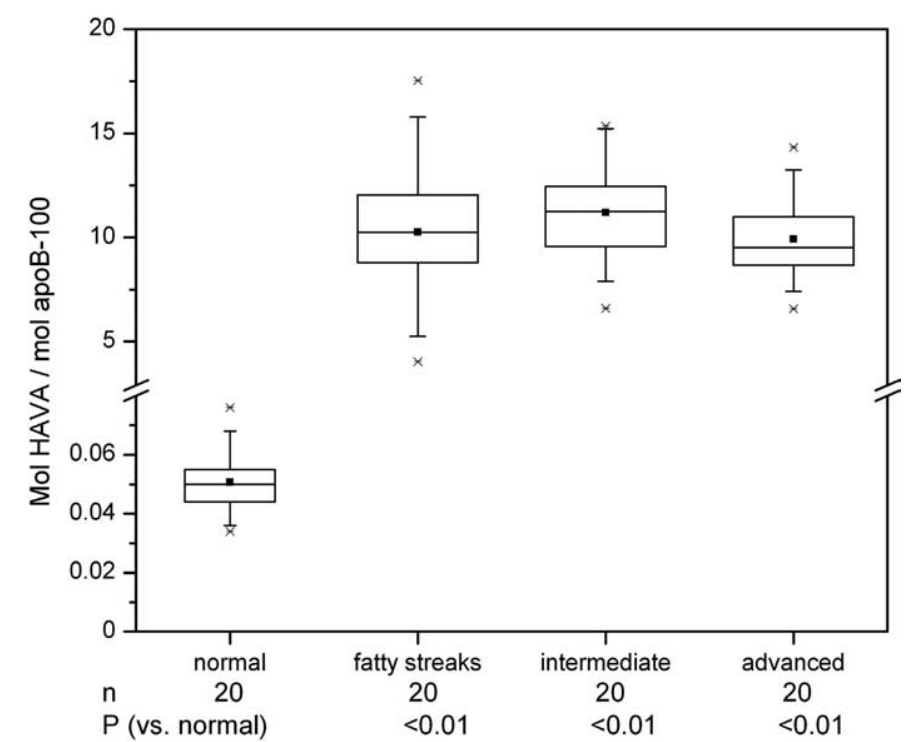

Fig. 1. Box plots showing low density lipoprotein apolipoprotein B-100 (apoB-100) 5-hydroxy-2-aminovaleric acid (HAVA) content in intima from various stages of lesion evolution in human thoracic aorta. 


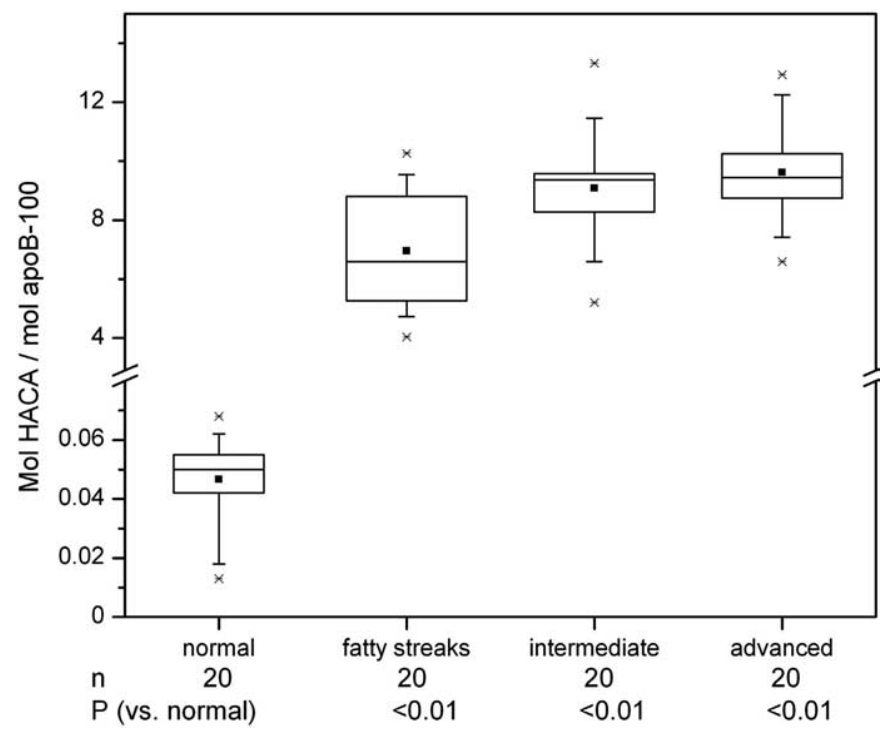

Fig. 2. Box plots showing low density lipoprotein apolipoprotein B-100 (apoB-100) 6-hydroxy-2-aminocaproic acid (HACA) content in intima from various stages of lesion evolution in human thoracic aorta.

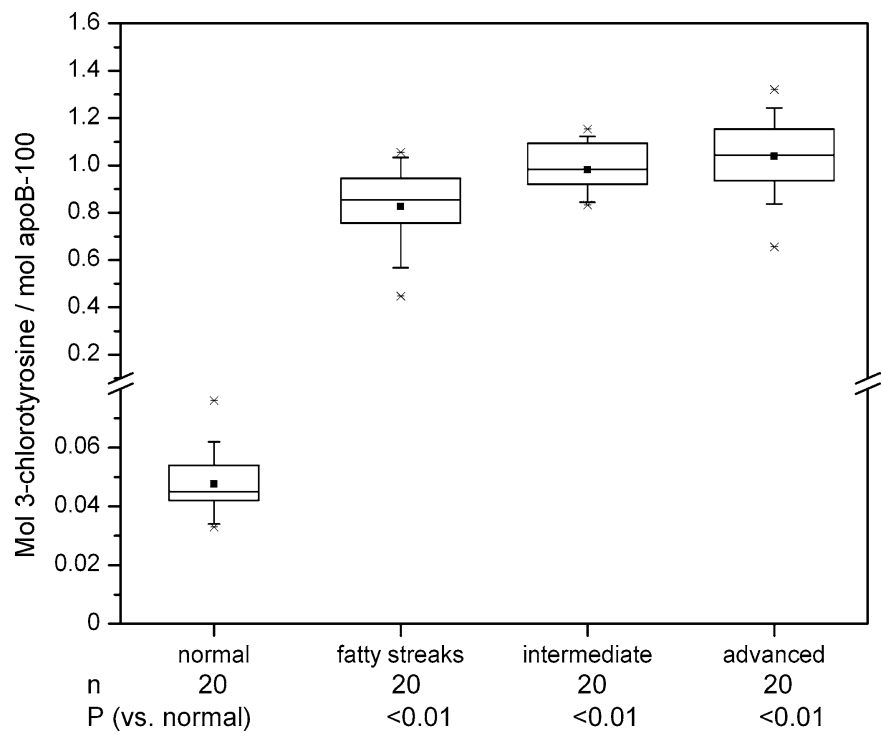

Fig. 3. Box plots showing low density lipoprotein apolipoprotein B-100 (apoB-100) 3-chlorotyrosine content in intima from various stages of lesion evolution in human thoracic aorta.

The content of all three oxidation products was significantly higher in LDL recovered from all types of lesion (including fatty streaks that represent the earliest lesion of atherosclerosis) when compared with normal aortic tissue (paired Student's $t$ test). These results suggest that metal catalyzed and MPO catalyzed processes contribute to LDL oxidation both early and late in the disease process. The mature apoB-100 consists of a single polypeptide chain of 4536 amino acids, and there is only one copy of the protein on each LDL particle. ApoB-100 ( $\mathrm{M}_{\mathrm{r}}$ 516,000; without carbohydrate content) contains 148 
arginine, 170 proline, 351 lysine, and 148 tyrosine residues [19]. The overall yield of HAVA and HACA, respectively, that has been found in lesion LDL (mean HAVA content: $10.45 \pm 0.66 \mathrm{~mol} / \mathrm{mol}$ apoB-100, equally to $328 / 10,000$ proline plus arginine residues; mean HACA content: $8.55 \pm 2.06 \mathrm{~mol} / \mathrm{mol}$ apoB-100, equally to $243 / 10,000$ lysine residues) is remarkably high and indicates that proline, arginine, and lysine residues are good targets for metal catalyzed oxidative attack. Furthermore, the high level of 3-chlorotyrosine in lesion LDL (mean 3-chlorotyrosine content: $0.94 \pm 0.17 \mathrm{~mol} / \mathrm{mol}$ apoB-100, equally to $64 / 10,000$ tyrosine residues) indicates that tyrosine residues are good targets for MPO catalyzed oxidation in atherosclerotic tissue. Control experiments indicated that sample processing is unlikely to be contributing to HAVA, HACA, and 3-chlorotyrosine formation as an artifact (data not shown in detail). The content of HACA, HAVA, and 3-chlorotyrosine in both atherosclerotic and normal aortic tissue exceeded the physiological level of HAVA $(0.012 \pm 0.004 \mathrm{~mol} / \mathrm{mol}$ apoB-100 $(0.4 / 10,000$ proline plus arginine residues)), HACA $\left(7.1 \times 10^{-4} \mathrm{~mol} / \mathrm{mol}\right.$ apoB-100 $(0.02 / 10,000$ lysine residues $\left.)\right)$, and 3 -chlorotyrosine $\left(3.3 \times 10^{-4} \mathrm{~mol} / \mathrm{mol}\right.$ apoB-100 $(0.02 / 10,000$ tyrosine residues $\left.)\right)$ found in native plasma LDL obtained from healthy normolipidemic, normoglycemic subjects $[6,10,18]$. Furthermore, the findings are consistent with former data showing increased HAVA content $(0.063 \pm 0.02 \mathrm{~mol} / \mathrm{mol}$ apoB-100 (2.0/10,000 proline plus arginine residues)) in circulating LDL recovered from hypercholesterolemic subjects who were at high atherosclerotic risk [20]. The experiments also support the hypothesis that pathways involving both metal catalyzed oxidation and myeloperoxidase catalyzed oxidation of LDL apoB-100 may be of pathological significance for atherogenesis [5,21,22]. However, additional work is needed to understand both the nature of the original oxidative insult and the specific consequences of $\gamma \mathrm{GSA}, \alpha \mathrm{ASA}$, and 3-chlorotyrosine formation for the metabolic fate of apoB-100-containing lipoproteins in vivo. The specificity and sensitivity of the GC/MS method employed make this a tool to get further insight into the contributions of oxidative damage to the onset and progression of atherosclerosis as well as other diseases.

\section{Acknowledgements}

The authors are grateful to Mrs. Sigrid Nitzsche from the Lipoprotein Laboratory, Department of Internal Medicine 3, and the staff of the Institute of Legal Medicine, Carl Gustav Carus Medical School, University of Technology Dresden, Germany, for expert technical assistance.

\section{References}

[1] J.W. Heinecke, Oxidized amino acids: culprits in human atherosclerosis and indicators of oxidative stress, Free Radic. Biol. Med. 32 (2002), 1090-1101.

[2] E.R. Stadtman, Importance of individuality in oxidative stress and aging, Free Radic. Biol. Med. 33 (2002), 597-604.

[3] J.A. Berliner and J.W. Heinecke, The role of oxidized lipoproteins in atherogenesis, Free Radic. Biol. Med. 20 (1996), 707-727.

[4] R. Ross, Atherosclerosis - an inflammatory disease, N. Engl. J. Med. 340 (1999), 115-126.

[5] S.L. Hazen and J.W. Heinecke, 3-Chlorotyrosine, a specific marker of myeloperoxidase-catalyzed oxidation, is markedly elevated in low density lipoprotein isolated from human atherosclerotic intima, J. Clin. Invest. 99 (1997), 2075-2081.

[6] J. Pietzsch, S. Kopprasch and R. Bergmann, Analysis of 3-chlorotyrosine as a specific marker of protein oxidation: The use of $\mathrm{N}(\mathrm{O}, \mathrm{S})$-ethoxycarbonyl trifluoroethyl ester derivatives and gas chromatography/mass spectrometry, Rapid Commun. Mass Spectrom. 17 (2003), 767-770.

[7] A.L.P. Chapman, M.B. Hampton, R. Senthilmohan, C.C. Winterbourn and A.J. Kettle, Chlorination of bacterial and neutrophil proteins during phagocytosis and killing of Staphylococcus aureus, J. Biol. Chem. 277 (2002), 9757-9762. 
[8] Y.I. Miller and N. Shaklai, Kinetics of hemin distribution in plasma reveals its role in lipoprotein oxidation, Biochim. Biophys. Acta 1454 (1999), 153-164.

[9] J.R. Requena, C.C. Chao, R.L. Levine and E.R. Stadtman, Glutamic and aminoadipic semi-aldehydes are the main carbonyl products of metal-catalyzed oxidation of proteins, Proc. Natl. Acad. Sci. USA 98 (2001), 69-74.

[10] J. Pietzsch, Measurement of 5-hydroxy-2-aminovaleric acid as a specific marker of iron-mediated oxidation of proline and arginine side-chain residues of low-density lipoprotein apolipoprotein B-100, Biochem. Biophys. Res. Commun. 270 (2000), 852-857.

[11] J. Pietzsch and U. Julius, Different susceptibility to oxidation of proline and arginine residues of apolipoprotein B-100 among subspecies of low density lipoproteins, FEBS Lett. 491 (2001), 123-126.

[12] M. Akagawa, T. Sasaki and K. Suyama, Oxidative deamination of lysine residue in plasma protein of diabetic rats. Novel mechanism via the Maillard reaction, Eur. J. Biochem. 269 (2002), 5451-5458.

[13] P. Cao and M. Moini, Quantitative analysis of fluorinated ethylchloroformate derivatives of protein amino acids and hydrolysis products of small peptides using chemical ionization-mass spectrometry, J. Chromatogr. A 759 (1997), 111117.

[14] M. Vatankhah and M. Moini, Characterization of fluorinated ethylchloroformate derivatives of protein amino acids using positive and negative chemical ionization gas chromatography/mass spectrometry, Biol. Mass Spectrom. 23 (1994), 277282.

[15] J. Pietzsch, U. Julius and M. Hanefeld, Stable isotope ratio analysis of amino acids: the use of N(O)-ethoxycarbonyl trifluoroethyl ester derivatives and gas chromatography/mass spectrometry, Rapid Commun. Mass Spectrom. 11 (1997), $1835-1838$.

[16] PDAY Research Group, Natural history of aortic and coronary atherosclerotic lesions in youth: findings from the PDAY study, Arterioscler. Thromb. 13 (1993), 1291-1298.

[17] J. Pietzsch, S. Subat, S. Nitzsche, W. Leonhardt, K.U. Schentke and M. Hanefeld, Very fast ultracentrifugation of serum lipoproteins: influence on lipoprotein separation and composition, Biochim. Biophys. Acta 1254 (1995), 77-88.

[18] J. Pietzsch and R. Bergmann, Analysis of 6-hydroxy-2-aminocaproic acid (HACA) as a specific marker of protein oxidation: The use of $\mathrm{N}(\mathrm{O}, \mathrm{S})$-ethoxycarbonyl trifluoroethyl ester derivatives and gas chromatography/mass spectrometry, Amino Acids 26 (2004), 45-51.

[19] J. Scott, The molecular and cell biology of apolipoprotein-B, Mol. Biol. Med. 6 (1989), 65-80.

[20] J. Pietzsch, P. Lattke and U. Julius, Oxidation of apolipoprotein B-100 in circulating low density lipoproteins is related to low density lipoprotein residence time: in vivo insights from stable isotope studies, Arterioscler. Thromb. Vasc. Biol. 20 (2000), E63-E67.

[21] J.W. Heinecke, Mass spectrometric quantification of amino acid oxidation products in proteins: insights into pathways that promote LDL oxidation in the human artery wall, FASEB J. 13 (1999), 1113-1120.

[22] M.J. Davies, S. Fu, H. Wang and R.T. Dean, Stable markers of oxidant damage to proteins and their application in the study of human disease, Free Radic. Biol. Med. 27 (1999), 1151-1163. 


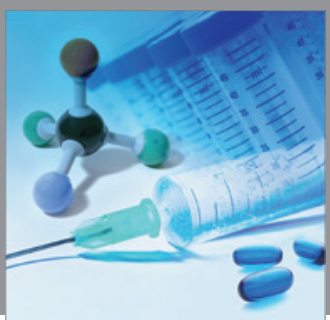

International Journal of

Medicinal Chemistry

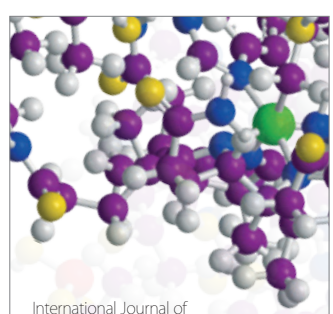

Carbohydrate Chemistry

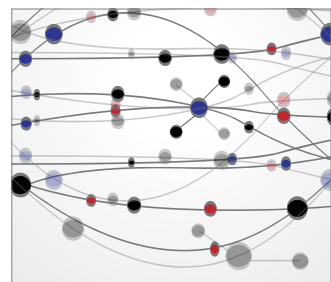

The Scientific World Journal
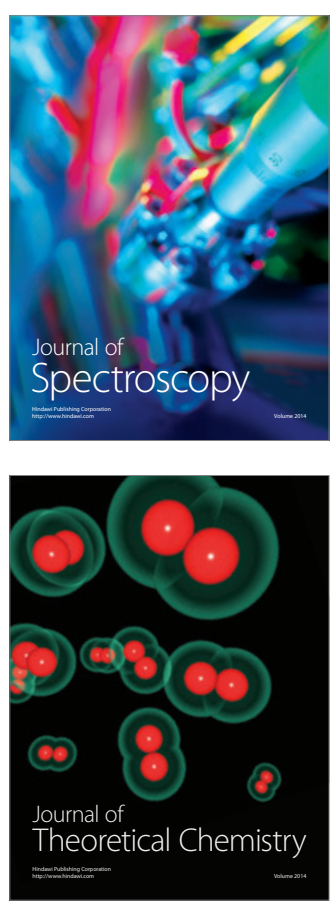
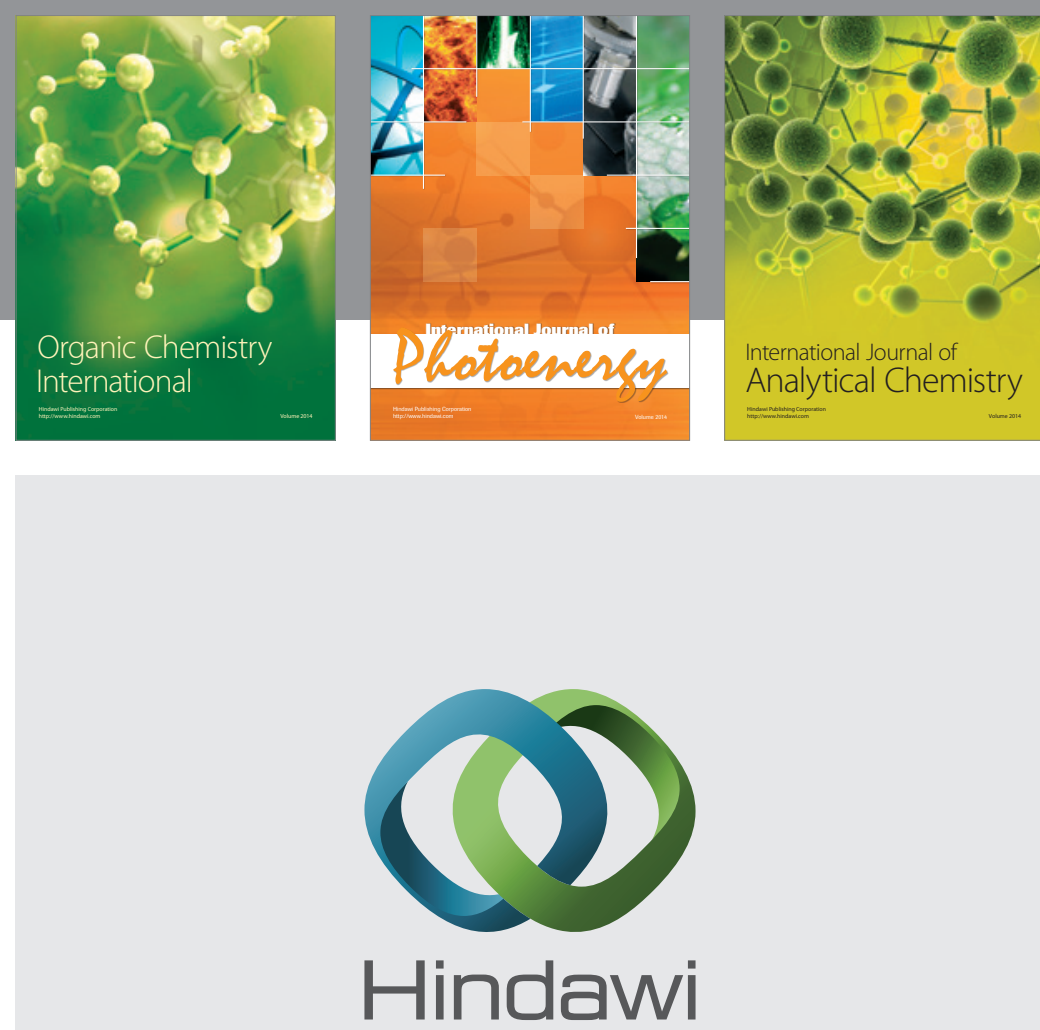

Submit your manuscripts at

http://www.hindawi.com
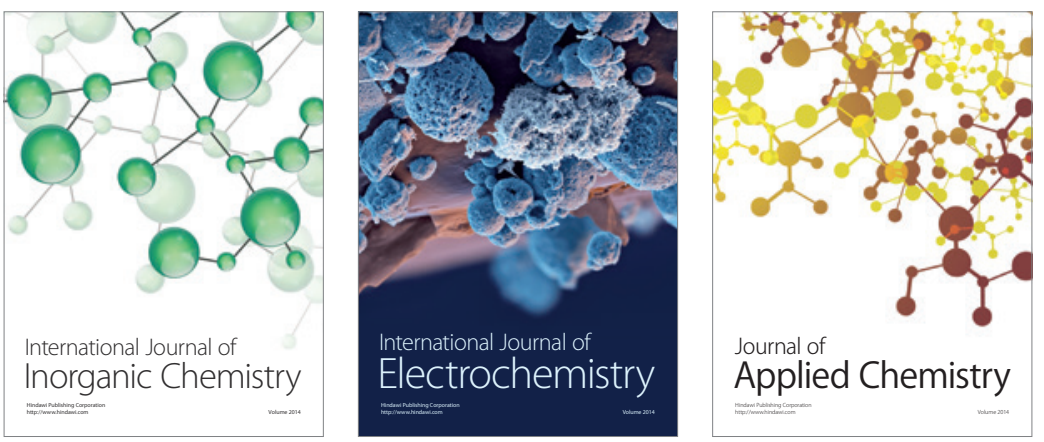

Journal of

Applied Chemistry
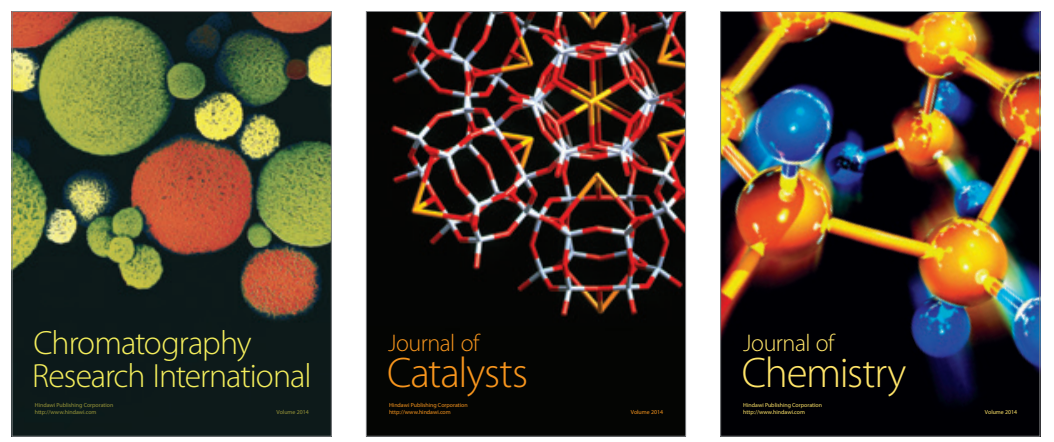
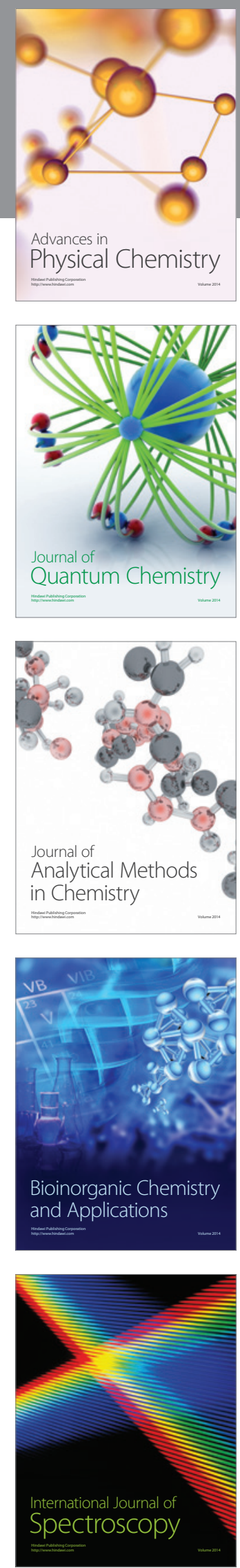